\title{
Computer Aided Kinematic Analysis of Toggle Clamping Mechanism
}

\author{
S.A. Bhojne ${ }^{1,2}$, A.A. Keste ${ }^{1}$, S.H. Gawande ${ }^{1}$, D.H. Thakur ${ }^{2}$ \\ ${ }^{I}$ (Department of Mechanical Engineering, M.E.S. College of Engineering, S.P. Pune University, Pune, India) \\ ${ }^{2}$ (John Deere India Pvt. Ltd., Pune, India)
}

\begin{abstract}
Toggle mechanism is used widely in the mechanical industry for various applications ranging from injection molding machines to tools \& fixtures. Toggle clamps are available for different types to suit desired application. This paper explores the kinematic analysis of the toggle clamping mechanism. Toggle mechanism considered here is actuated by hydraulic cylinder. Position, velocity and acceleration analysis is described in this work, for this analysis toggle clamping mechanism is fragmented into two basic mechanisms, Slider-Crank mechanism and Four-Bar Mechanism. Slider-Crank generates torque and output force in the toggle clamp mechanism so emphasis is given on slider-crank mechanism in this work. Toggle clamp mechanism for workpiece bending operation is described in this work. Mechanical advantage of the linkage is the key parameter for linkage performance, mechanical advantage and it's relation with torque and link parameter is discussed in brief.
\end{abstract}

Keywords - Toggle Mechanism, Analytical study, CADAnalysis.

\section{INTRODUCTION}

Toggle clamping mechanism is widely used in the manufacturing industry in the areas like bending, grinding, welding, fitting etc. Tools \& fixtures industry are the major user of the toggle clamp mechanism, metal working and assembly are the areas where this mechanism is seen often. Toggle mechanisms are commonly used within linkages in order to lock their position. These mechanisms do this by passing through a "toggle" point where any force into the linkage would cause the linkage to maintain its position. While passing through the "toggle" point, there is a great deal of stress applied to the links. . The purpose of this investigation was to evaluate the impact that a change in length of the links or change in the choice of material for the compliant element within the linkage has on the stress within the linkage. Ming-Shyan Haung et.al [1] explained that toggle mechanism can generate significantly larger clamping force for the given input force this is a key feature which makes this mechanism one of the favorites clamping mechanisms. Pei-Lum Tso [2] advised that $s$ good clamping fixture must be capable of overcoming the maximum possible force exerted by the work-piece and should be easy to operate, positioned, or loosened. Burton [3] explains hinge friction in the toggle mechanism. Beer and Johnston [4] used virtual work principle to conduct analysis of toggle vise. Mechanisms with more than four links are generally fragmented into basic linkages for simplifying the analysis of the overall mechanism. Four bar linkage and Slider - Crank linkage are the most favorite basic linkages for toggle mechanism fragmentation, converting toggle mechanism into four bar linkage and slider crank linkage will simplify the overall linkage and analysis can be done with ease [5, 6]. Lin and Kuo [7] worked out the thrust requirement for mold-clamping process of five point double toggle clamping mechanism; they also put forward the formula to evaluate thrust of cross head mechanism for given clamping force. Fung et al. [8] applied Hamilton's principle and Lagrange's multiplier method to evaluate an expression for the inertial force and the mechanism thrust. The Runge-Kutta integral method was utilized to explore the kinematics and dynamics and compare them with four-point and five-point toggle mechanisms. Fung et al. [9] examined the kinematics sensitivity of a toggle mechanism, which is considered as a combination of two slider-crank mechanisms, and shown better clamping efficiency. To decrease the friction force, Fung et al. [10] put forward a new presentation of the toggle mechanism which is operated by a controlled permanent magnet and linear synchronous motor instead of gears and other additional mechanisms. Toggle clamp mechanism is a classic example of mechanical advantage utilization. Manual operated toggle clamp are most popular in small and mid-scale industries to be utilized for fixtures while machining and bending operations. The toggle clamps have the advantage of their favorable power to movement ratio, and easy action.

A generic toggle mechanism can be considered as a good mix of couple of traditional linkages, a fourbar linkage and a slider-crank mechanism. So to conduct the analysis of entire toggle mechanism two separate 
linkages are to be studied separately and the outcomes to be combined. This work focuses mostly on output mechanism which is a slider-crank linkage.

\section{SYSTEM DESCRIPTION}

are;

Fig. 1 shows the toggle clamping mechanism. Ground link is represented by link $\mathrm{C}$ and other details

Element Details;

A - Clamping member (output link)

$\mathrm{P}$ - Input link (Hydraulic cylinder)

$\mathrm{C}-$ Ground link

$\mathrm{m}-$ Coupler (Output link)

$\mathrm{n}-$ Crank (Support link)

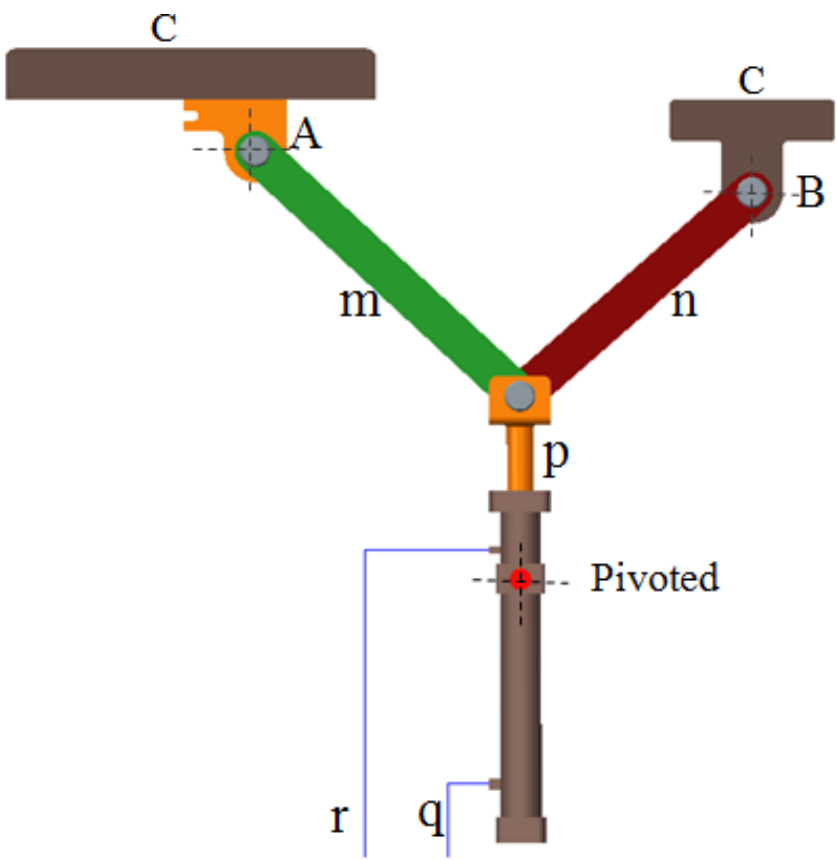

Fig. 1: Toggle Clamping Mechanism

Input velocity and hence input force is generated by the virtue of hydraulic system pressure. Hydraulic cylinder is working as an actuator for the overall toggle clamping system. Clamping member, A is an output link which holds the clamping jaw. Output link velocity and the force available at the output link which holds or clamp the work piece is a function of the mechanism configuration, link position, link lengths, joint friction and actuator parameters (system pressure, hydraulic cylinder specifications).

\section{DESIGN PARAMETERS}

The main objective of the toggle clamping mechanism design is to generate a maximum mechanical advantage and kept the cost low. Design parameters of the toggle clamp mechanism mentioned below are crucial in formulating the optimization function of the mechanism. This optimization function can solved using different optimization techniques such as genetic algorithm or artificial neural network.

Following parameters are important for toggle clamping mechanism design:

1. Velocity of the clamping/sliding member

2. Stroke and velocity of hydraulic cylinder

3. Force magnification factor

4. Pin reaction at joints

5. Initial position of the links

6. Structural strength

1. Velocity of the clamping member is a function of linkage position, input velocity of hydraulic actuator, frictional effect in sliding members and pin joints 
2. Stroke and velocity of hydraulic actuator can be set as required hydraulic pressure can be adjusted and fine tuned at initial setting. Selection of actuator is depend upon the force requirement for mechanism operation

3. Force magnification factor is the key feature for the design and optimization of the toggle clamp mechanism, mechanical advantage plays important role minimize the input force and to maximize the output force

4. Pin reaction at joints is also important parameter to be considered for the performance of the toggle clamp mechanism

Toggles clamping mechanism is utilized in the manufacturing industry to support the processes such as clamping, pressing, bonding etc. So it is a need of an industry to have a toggle mechanism which generates maximum output with minimum input efforts. Mechanical advantage relates the output torque and input torque it also relates the link lengths as descried below. Mechanical advantage is the ratio of output torque to the input torque. The mechanical advantage $M a$ is expressed as;

$$
M a=\frac{T o}{T i}=\frac{\text { Output torque of toggle clamp }}{\text { Input torque of the mechanism }}
$$

The ratio between actuating input force $F_{i}$ and output clamping force $F_{o}$ is given in terms of mechanical advantage by [2];

$$
\begin{gathered}
\frac{F_{0}}{F_{i}}=\text { Ma } \frac{l_{i}}{l_{0}} \\
\frac{l_{i}}{l_{0}}=\frac{\text { Length of Crank }}{\text { Lenght of Coupler }}
\end{gathered}
$$

Toggle clamping mechanisms is one of the classic examples of the mechanism which utilizes the mechanical advantage. Emphasis is given on generating the maximum torque or movement at the output member with the given input, considering constraints on space available. Input force generated by the actuator is transmitted to the coupler link via pin joints, consideration of pin joint design, material of pin and overall friction in the joint are some of the important considerations in the design of the toggle clamp linkage. Locking of the mechanism is avoided by constraining the joints appropriately and it is ensured that the minimum angle to be maintained between coupler link and crank. The instant when the angle between coupler link and crank becomes $180^{\circ}$, toggle mechanism get locked. So it is necessary to maintain a minimum angle between these two links in the fully extended condition.

Material selection is one the key aspects in design of any machine components, considering working conditions of the toggle linkages coupler link and crank will experience tensile as well as compressive loads, pin locations are subjected to the shear load.

\section{Analytical STUdY}

Toggle clamping mechanism can be fragmented in two basic mechanisms, Slider-Crank linkage and Four Bar linkage. Slider-Crank mechanism is applied to perform analysis on clamping member and Four-Bar mechanism is applied for analysis of input system. In this study analysis of output system conducted. Fig. 2 shows the CAD model of toggle mechanism considered for the analytical study as well as computer aided synthesis of the mechanism.

Point "A" represents the clamping member, point B and $\mathrm{P}$ represents ground pivot and mechanism actuator respectively. Mechanism actuator, hydraulic cylinder is operated by hydraulic system, q \& $\mathrm{r}$ represents the supply line and return line respectively to operate the hydraulic cylinder.

Links $\mathrm{m}$ and $\mathrm{n}$ are representing the coupler and crank respectively. Crank is pivoted to the ground link while coupler link is connected to the clamp member. Several other applications along with clamping could be derived from this configuration of mechanism such as pressing, punching, bonding etc. Scope of this study is to consider the link " $m$ " and point "A" for the analysis.

Position, Velocity and Acceleration analysis is performed to obtain the response of the given input on the output link $\mathrm{m}$ and intern on the point A. velocity and acceleration analysis are conducted in time domain environment to check the closeness with the CAD software results and conduct verification study. Section below describes all the three analysis in detail.

Analytical study on the toggle clamping linkage is performed in time domain at specific steps as an interval. At each time step the input and output quantities are collected together and plotted. 
Analytical Procedure: Fig. 3 shows the geometric details for analytical study. Angle $\beta$ is the angle made by clamp member with the coupler link considering larger face of the clamp member remains parallel to the ground. Crank makes an angle $\varphi$ with each ground.

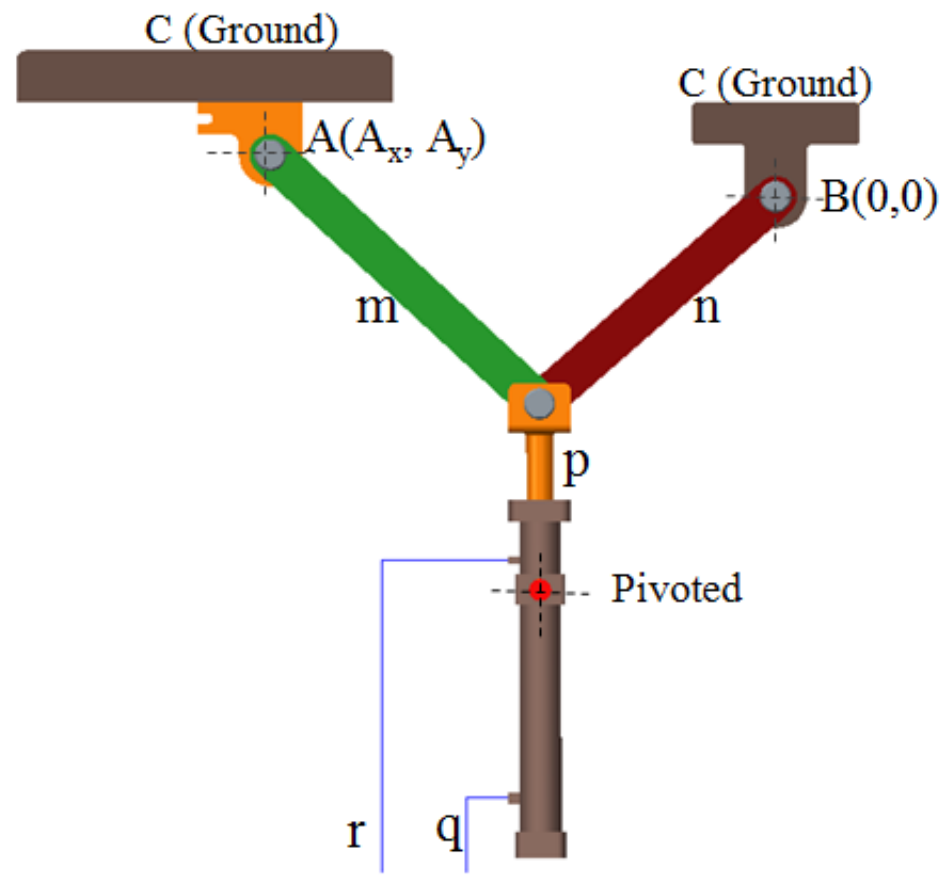

Fig.2 : Toggle Clamp Mechanism

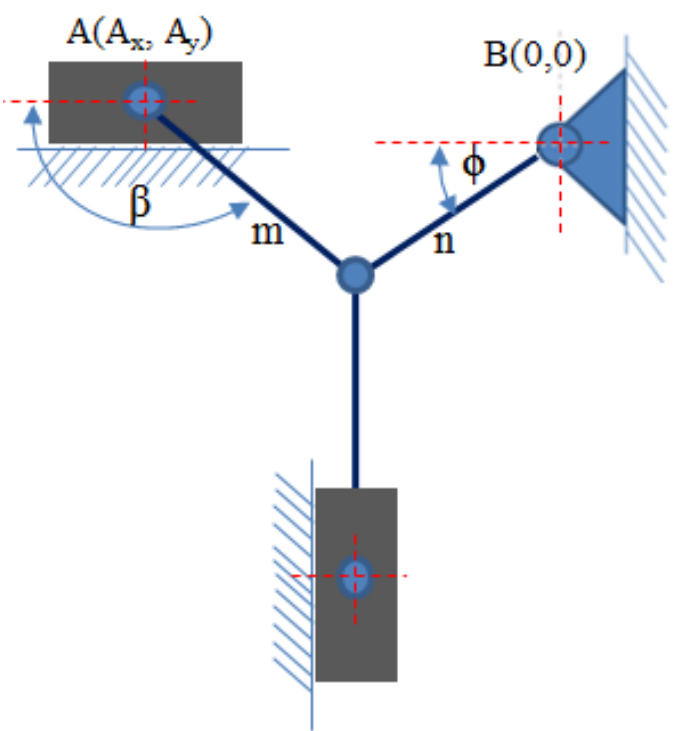

Fig. 3 : Position Analysis of Toggle Clamp

Equation 1 represents the position of the clamp member at any instance, angle $\square$ is the input variable and angle $\beta$ is to be evaluated based on the initial condition and geometry of the clamping mechanism.

$$
A x=n \cos \emptyset-m \cos \beta
$$

Differentiating equation 1 w.r.t. time, we get the velocity of A. Equation 2 denotes velocity of clamp member.

$$
V_{A}=-n \phi^{\prime}(\sin \emptyset)-m \beta^{\prime}(\cos \beta)
$$

Differentiation of above equation 2 w.r.t. time gives acceleration of point A, equation 3 denotes acceleration. 


$$
A_{A}=-\phi^{n} n(\cos \emptyset)-m \phi^{\prime 2}(\cos \phi)+m \beta^{n}(\sin \beta)+m \beta^{2}(\cos \beta)
$$

Table 1 shows the dimensional details.

Table 1 : Dimensional Details

\begin{tabular}{|c|c|c|c|c|}
\hline Sr. No. & Measure & Value & Unit & Description \\
\hline 1 & $\mathrm{~m}$ & 175 & $\mathrm{~mm}$ & Coupler link \\
\hline 2 & $\mathrm{n}$ & 150 & $\mathrm{~mm}$ & Crank link \\
\hline 3 & $\square$ & 41 & Deg. & Crank angle \\
\hline 4 & $\beta$ & 137 & Deg. & Crank angle \\
\hline
\end{tabular}

\section{CAD MODELLING AND ANALYSIS}

Toggle clamping mechanism discussed is analyzed using mechanism package of CAD software. Fig. 4 explains the generalized procedure followed for conducting a computer aided analysis of mechanism.

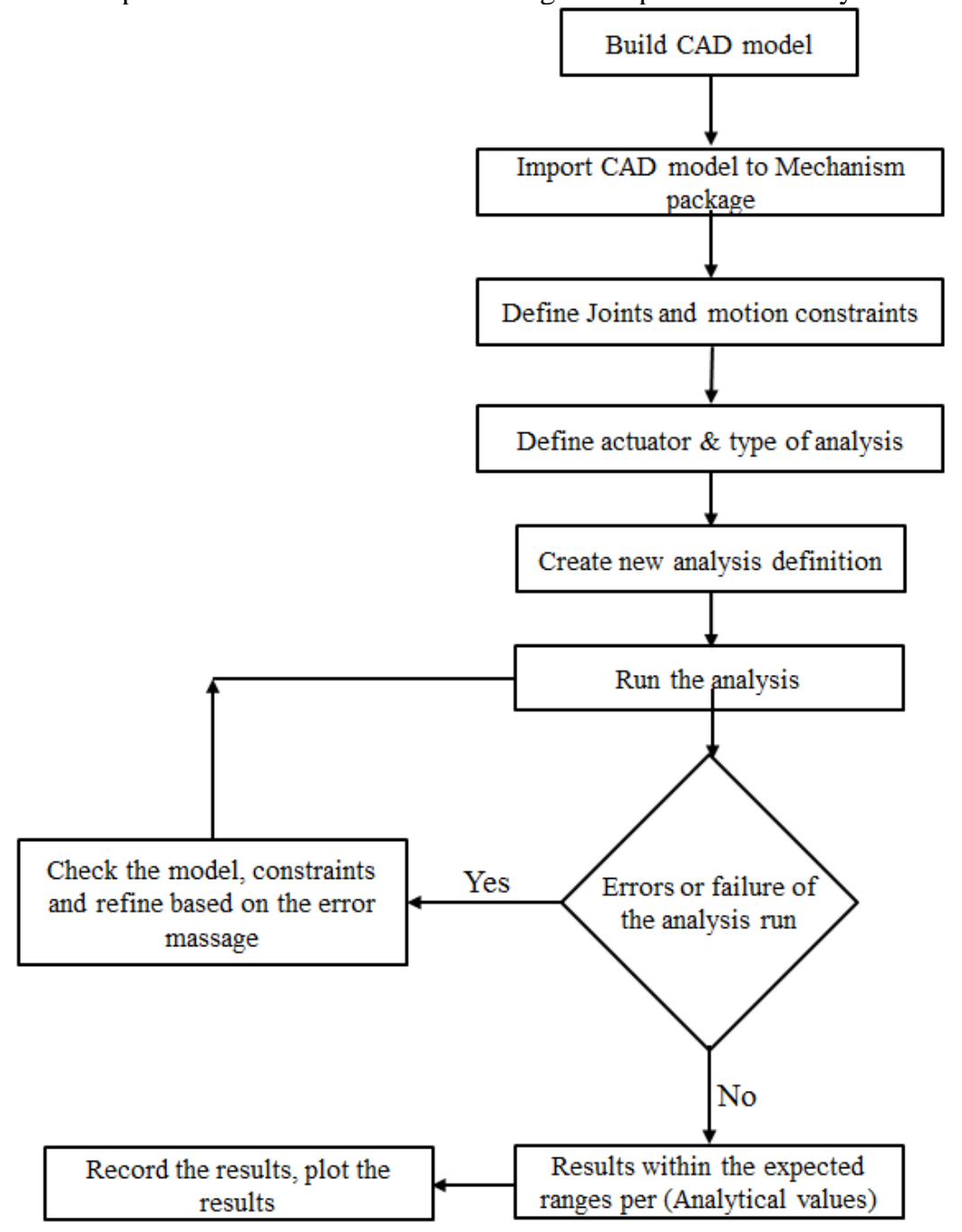

Fig.4 : Mechanism Analysis Procedure

CAD model can be defined in few of the mechanism packages else model need to be imported from the solid modelling package to mechanism package. Post receiving the required model in the mechanism package it is very important to define the joints between different links. All the joints definition required to constraints the motion are available in the $\mathrm{CAD}$ packages, pin, cylinder, slider are some the most useful joints. After defining the joints, motion constraints are applied. Actuators such as motor, cylinder are connected at required junctions. Further to this, new analysis definition is created and analysis is run for the applied constraints and actuator 
configuration. If there are no errors and warning are observed; results can be plotted in the required format and can be used for further study and review. If errors are observed, model need to be refined and constraints as well as actuator configuration to be verified and run the model. Interpolation method can be used to correlate the initial condition between software values and theoretical calculations. Fig. 5 shows the initial position and extended position of the toggle clamping mechanism used for analysis.

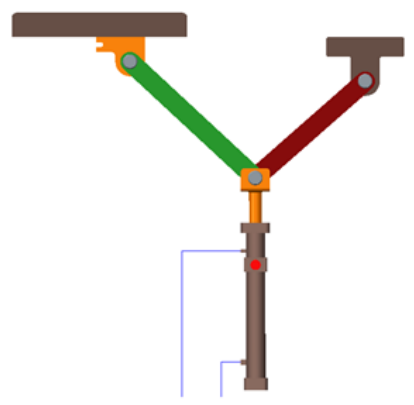

Initial Position

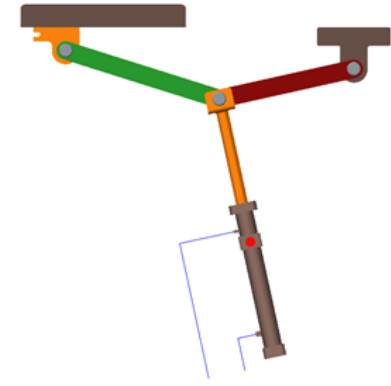

Extended Position

Fig.5 : Computer aided analysis of toggle clamp

Model simulation is conducted using mechanism package of the CAD software. Model parameters are set at initial condition and time step inputs are given to actuator to attain the required extended position.
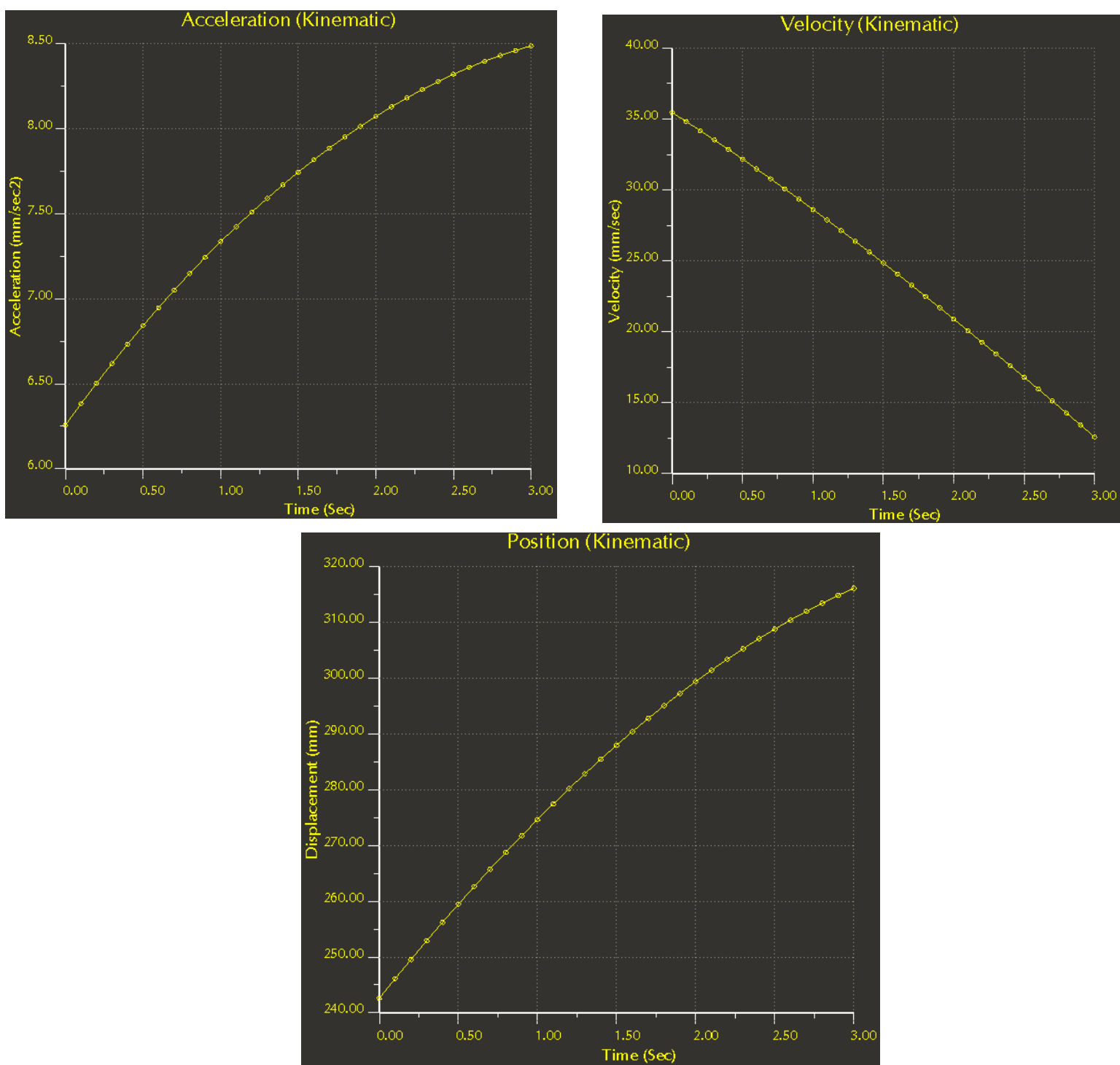

Fig. 6: CAD software results of kinematic analysis 
Fig.6 shows the graphs from kinematic analysis of CAD model. Position, velocity and acceleration analysis is performed and results are plotted on time domain. Analysis was run for specific time frame and data is recorded at specific time interval.

\section{RESULTS AND DISCUSSION}

Analytical results are compared with the CAD software results and graphs are plotted for position, velocity and acceleration analysis. Fig.7 represents the position analysis; it is observed that displacement of point A is linear with time. Initial distance between coordinate system and point A along negative X-axis is $241 \mathrm{~mm}$. Crank angle is modified at an interval of 0.5 second each and position of $\mathrm{A}$ is noted at each interval. After 3 seconds, point A reached at extended position, at this instant point A reached at $315 \mathrm{~mm}$ from coordinate system. In similar way velocity and acceleration of point A is noted. Fig. 8 and Fig.9 shows the velocity and acceleration analysis results.

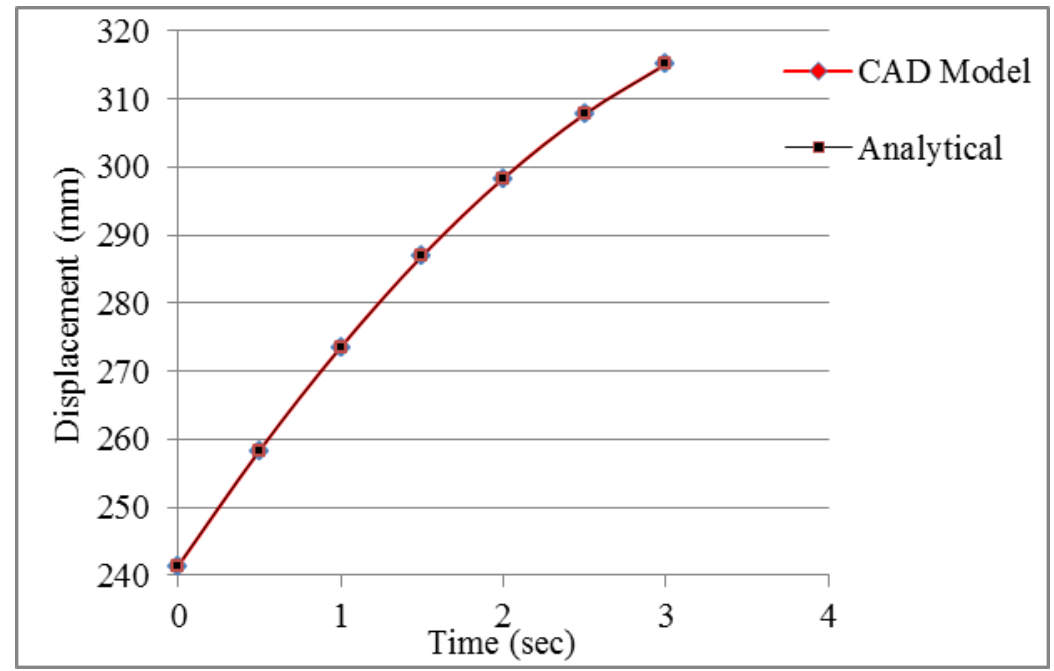

Fig.7: Position Analysis

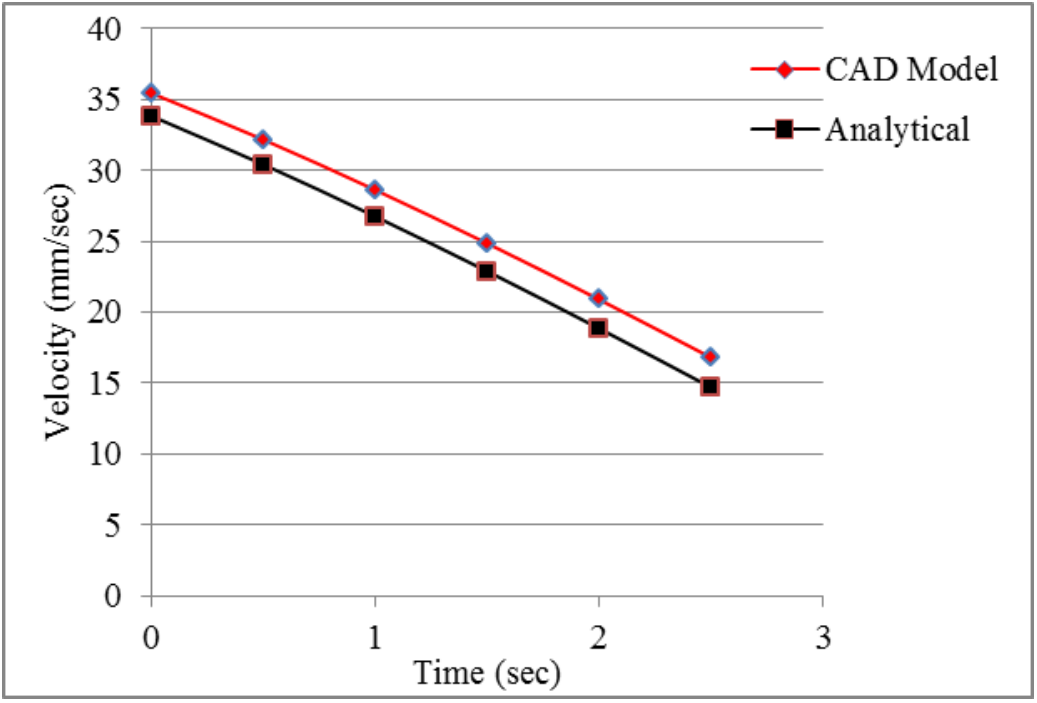

Fig 8: Velocity Analysis

For velocity and acceleration analysis the trends of the analytical results are matching with the CAD software results. 


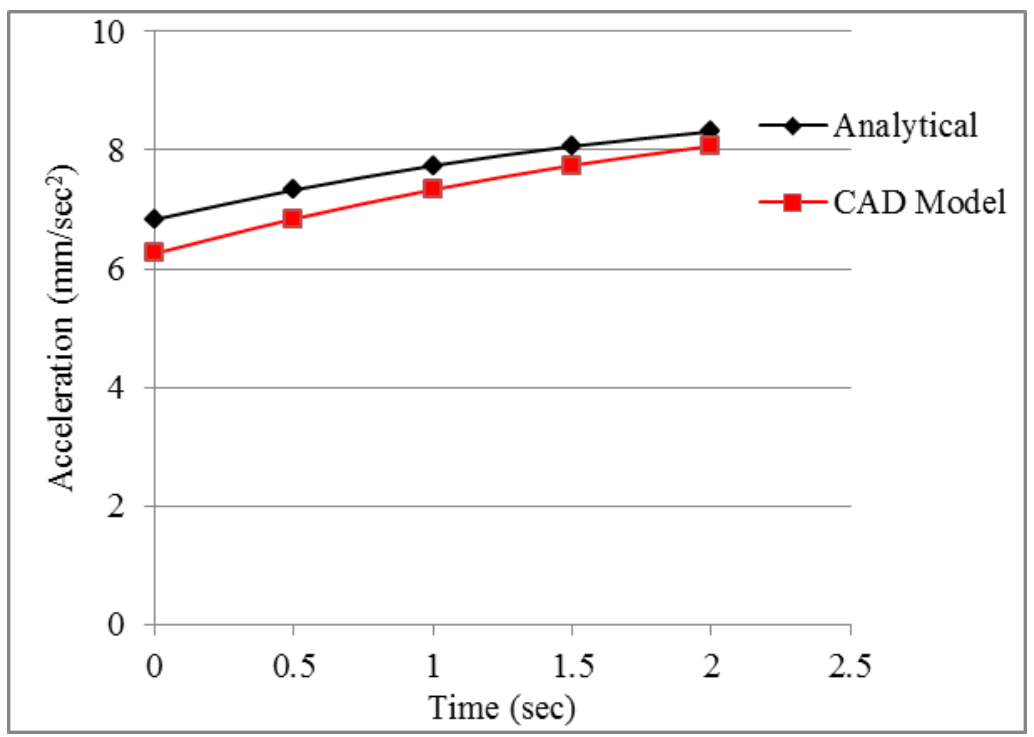

Fig.9: Acceleration Analysis

VII. CONCLUSION

The objective of this work is to study the effect of dimensional parameter (crank angle) on coupler link. This objective is achieved by performing analytical and numerical analysis for position, velocity and acceleration. Numerical and analytical results are studied and plotted for validating the degree of closeness. It is observed that the trends of analytical solution and software results are matching closely. From position analysis; it is observed that displacement of point A is linear with time. Initial distance between coordinate system and point A along negative $\mathrm{X}$-axis is $241 \mathrm{~mm}$. Crank angle is modified at an interval of 0.5 second each and position of A is noted at each interval. After 3 seconds, point A reached at extended position, at this instant point A reached at $315 \mathrm{~mm}$ from coordinate system. The methodology used for analytical study is verified with the CAD software package. Computation of optimization function and optimization of toggle clamp mechanism will be the future scope of this study.

\section{REFERENCES}

[1] Ming-shyan Huang et.al, Key design parameters and optimal design of a five-point double-toggle clamping mechanism, Applied Mathematical Modelling 35(2011) 4304-4320.

[2] Pei-Lum Tso, The kinematic synthesis of toggle clamps, ASME,Journal of manufacturing science and engineering Aug 1998 Vol. $120 / 649$.

[3] P. Burton, Kinematics and Dynamics of Planar Machinery, Prentice Hall, Englewood Cliffs, NJ, 1979.

[4] F.P. Beer, E.R. Johnston, Vector Mechanics for Engineers - Statics, McGraw Hill, New York, 1984.

[5] G.H. Martin, Kinematics and Dynamics of Machines, McGraw Hill, New York, 1982.

[6] C.E. Wilson, J.P. Sadler, Kinematics and Dynamics of Machinery, Harper Collins College Publishers, 1993

[7] W.Y. Lin, M.H. Kuo, Investigation of the friction effect at pin joints for the five-point double-toggle clamping mechanisms of injection molding machines, International. Journa. Mechanical Science. 45, (2003,) 913-1927.

[8] R.F. Fung, C.C. Hwang, C.S. Huang, W.P. Chen, Inverse dynamics of a toggle mechanism, Comput. Struct. 63 (1997) 91-99.

[9] R.F. Fung, C.C. Hwang, C.S. Huang, Kinematic and sensitivity analyses of a new type toggle mechanism, Jpn. Soc. Mech. Eng. Ser. C 40, (1997.) 360-365.

[10] R.F. Fung, J.W. Wu, D.S. Chen, A variable structure control toggle mechanism driven by a linear synchronous motor with joint coulomb friction, J. Sound Vib. 247 (2001), 741-753. 